\title{
openheart Association of QT interval with mortality by kidney function: results from the National Health and Nutrition Examination Survey (NHANES)
}

\author{
Rehan Malik, ${ }^{1,2}$ Sehrish Waheed, ${ }^{3}$ Deepak Parashara, ${ }^{4}$ Jorge Perez, \\ Salman Waheed ${ }^{4}$
}

To cite: Malik R, Waheed S, Parashara D, et al. Association of QT interval with mortality by kidney function: results from the National Health and Nutrition Examination Survey (NHANES). Open Heart 2017;4:e000683. doi:10.1136/ openhrt-2017-000683

Received 6 July 2017 Revised 16 August 2017 Accepted 22 August 2017

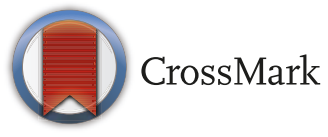

${ }^{1}$ Department of Medicine, Mount Sinai Medical Center, Miami, Florida, USA

${ }^{2}$ Center for Healthcare Advancement \& Outcomes Research, Miami, Florida, USA ${ }^{3}$ Good Shepherd Health System, University of Texas Health Science Center at Tyler, Longview, Texas, USA ${ }^{4}$ Department of Cardiology, University of Kansas Hospital, Kansas City, Kansas, USA

Correspondence to Dr Salman Waheed; salmanwaheed@hotmail.com

\section{ABSTRACT}

Objective Prolonged QT interval predisposes to ventricular arrhythmias and sudden cardiac death. However, the association between QT interval and mortality by the level of pre-existing kidney function has not been investigated.

Methods We followed 6565 participants from the National Health and Nutrition Examination Survey III for a median of 13.3 years. Sample divided according to corrected QT (QTc) interval was as follows: normal (QTc $<450 \mathrm{~ms}$ for men and $<460 \mathrm{~ms}$ for women) or prolonged. It was further categorised as follows: (1) no chronic kidney disease (CKD), that is, albumin to creatinine ratio (ACR) $<30 \mathrm{mg} / \mathrm{g}$ and estimated glomerular filtration rate (eGFR) $\geq 60 \mathrm{~mL} / \mathrm{min} / 1.73 \mathrm{~m}^{2}$; (2) CKD by eGFR only (eGFR $<60 \mathrm{~mL} /$ $\mathrm{min} / 1.73 \mathrm{~m}^{2}$, ACR $<30 \mathrm{mg} / \mathrm{g}$ ); (3) CKD by ACR only (ACR $>30 \mathrm{mg} / \mathrm{g}$, eGFR $>60 \mathrm{~mL} / \mathrm{min} / 1.73 \mathrm{~m}^{2}$ ) and (4) CKD by both. Cox proportional hazards models were used.

Results CKD group had prolonged QTc than those without CKD $(20.5 \%$ vs $12.9 \%, p<0.0001)$. Both prolonged QTc and CKD are independently associated with increased risk of mortality. When combined, risk of mortality is higher in those with CKD by eGFR with prolonged QTc than normal QTc (HR 2.6 (1.7-3.9) and 3.1 (1.7-5.4) vs 1.4 (1.1-1.7) and 1.7 (1.3-2.1) for all-cause and CV mortality). There is no significant difference in risk in those with CKD by ACR when QTc is prolonged. There is significant improvement in risk prediction for all-cause and CV mortality when QTC is added to CKD beyond established CV risk factors (net reclassification index $p<0.00001$ ).

Conclusion A screening ECG in those with CKD may help in finer risk stratification and may be considered.

\section{INTRODUCTION}

Chronic kidney disease (CKD) is a major public health problem with an estimated prevalence of $13 \%$ in the USA. ${ }^{1}$ According to the National Kidney Disease Education Program, the prevalence of CKD has more than doubled between 2000 and 2008 for individuals 60 years or older. ${ }^{2}$ CKD is the ninth leading cause of death, and killing more every year. ${ }^{3}$ Mortality from CKD is more often from cardiovascular disease-related complications than progression to ESRD. ${ }^{4}$ Studies have

\section{KEY QUESTIONS}

What is already known this subject? The association between prolonged QT interval and mortality has been examined. Prolonged QT is associated with increased mortality risk. Little is known about the association of QT interval with mortality in those with chronic kidney disease.

What does this study add?

Those with chronic kidney disease are more likely to have prolonged corrected QT than those without. Risk of all-cause and cardiovascular mortality is higher in those with chronic kidney disease (by estimated glomerular filtration rate) and prolonged corrected QT than those with normal corrected QT. Combining corrected QT interval and chronic kidney disease improves risk prediction for all-cause and cardiovascular mortality.

How might this impact on clinical practice? Further studies are needed to confirm these findings. Screening for prolonged corrected QT interval in those with chronic kidney disease may help in identifying those at increased risk of adverse events.

suggested that corrected QT (QTc) interval is longer in individuals with CKD than those without CKD. ${ }^{5}$ Prolonged QTc is associated with ventricular arrhythmias, sudden cardiac death and all-cause mortality. ${ }^{6-9}$ However, there are limited data on the joint association of CKD, QTc and their interaction with mortality. There is an interest in assessing the role of screening ECG in predicting risk of adverse outcomes in patients with CKD. ${ }^{1}$ More needs to be known about the joint association of CKD and QTC and their interaction with mortality. It is also important to examine if risk prediction for mortality improves when QTc is added to CKD. In this study, we aim to examine the joint association of CKD and QTc with risk of all-cause and cardiovascular mortality in a nationally representative 
sample of the US population. We also aim to examine if adding QTc to CKD improves risk prediction for all-cause and cardiovascular mortality.

\section{METHODS}

\section{Study population}

The National Health and Nutrition Examination Survey (NHANES) is a cross-sectional, multistage, stratified, cluster probability sample representing non-institutionalised civilian population of the USA. For this study, we used data from NHANES III, which was conducted during the periods of 1988-1994 by the National Center for Health Statistics. ${ }^{11}$ The National Center for Health Statistics of the Centres for Disease Control and Prevention institutional review board approved the protocol for NHANES III. All participants gave written informed consent. In NHANES III, 12-lead ECG was performed for subjects aged 40 years or older $(n=8561)$. We included those with available data on QT interval, serum creatinine and albumin to creatinine ratio (ACR) $(n=7939)$. We excluded those with QRS duration $>120 \quad(n=513)$ or missing data on any of the key variables. Our final sample size was 6565 .

\section{Data collection}

NHANES used a standardised questionnaire to collect data on demographic characteristics, educational level, smoking status, alcohol consumption, medical history and medication use. ${ }^{12}$ Detailed physical examination was performed at a mobile examination centre. Blood pressure was measured three times during home interview and three times during the physical examination. Laboratory test included high-density lipoprotien, total cholesterol, plasma glucose, serum $\mathrm{K}^{+}$, serum $\mathrm{Ca}^{2+}$ and $\mathrm{Mg}^{2+}$ levels. QT-prolonging medications were defined according to the Arizona Center for Education and Research on Therapeutics database. ${ }^{13}$

\section{Assessment of exposure and outcome}

Standard 12-lead resting ECG recordings were performed at the mobile examination centres using the Marquette MAC 12 (Marquette Medical Systems, Milwaukee, Wisconsin, USA). All ECGs were reviewed at the Central ECG Laboratory at Wake Forest University, WinstonSalem, North Carolina. Recordings were excluded if there were technical errors or inadequate quality. Dalhousie ECG analysis programme was used, and a representative P-QRS-T cycle was derived automatically by selective averaging. ${ }^{14}$ After obtaining QT interval and heart rate from the resting ECG, we used Bazett's formula to calculate QTc interval, as this is the most widely used formula in clinical practice. ${ }^{15}$

Serum creatinine was measured using a kinetic Jaffe method and recalibrated to standardised creatinine measurements obtained at the Cleveland Clinic Research Laboratory, as previously described [ (standard creatinine $=(0.960 \times$ serum creatinine $)-0.184)] .{ }^{16}$ CKD-EPI equation was used to estimate GFR. ${ }^{17}$ CKD by creatinine was considered to be present when eGFR was $<60 \mathrm{~mL} / \mathrm{min} /$ $\mathrm{m}^{2}$. Urine albumin level was measured by solid-phase florescence immunoassay, Coulter Synchron AS/Astra Analyzer (Beckman Coulter, Fullerton, California, USA). We defined CKD by ACR as ACR $\geq 30 \mathrm{mg} / \mathrm{g}$.

Participants were followed through 31 December 2006 for all-cause and cardiovascular mortality. Vital status was based on matching NHANES III with the national death index death certificate records. Mortality ascertainment is performed using probabilistic matching between NHANES III and national death index, as described previously. ${ }^{18}$ Cause of death was determined based on the underlying cause listed on the death certificate. The International Statistical Classification of Diseases, Ninth Revision (ICD-9) was used to classify death through 1999, and ICD-10 was used for deaths from 1999 onwards. Cardiovascular mortality was defined as mortality due to ICD-9 codes $390-434$ and $436-459$ or ICD-10 codes I00 to I99.

\section{Statistical analysis}

ECG sampling weights were used to account for the complex survey design. ${ }^{14}$ T-test and analysis of variance were used for continuous variables and $\chi^{2}$ for categorical variables. We used multivariate linear regression to examine the association of QTc with CKD adjusting for age, gender, race, education, income, diabetes, hypertension, history of cardiovascular disease, smoking, alcohol use, QT-prolonging medications and beta-blockers. We divided the sample into two categories by QTc (normal when QTc $<450 \mathrm{~ms}$ for men and $<460 \mathrm{~ms}$ for women or prolonged). We divided the sample into additional categories by CKD (no CKD when eGFR $>60 \mathrm{~mL}$ / $\mathrm{min} / \mathrm{m}^{2}$ and ACR $<30 \mathrm{mg} / \mathrm{g}$, CKD by eGFR only when eGFR $<60 \mathrm{~mL} / \mathrm{min} / \mathrm{m}^{2}$ and ACR $<30 \mathrm{mg} / \mathrm{g}$ and CKD by ACR only when ACR $>30 \mathrm{mg} / \mathrm{g}$ and eGFR $>60 \mathrm{~mL} / \mathrm{min} /$ $\mathrm{m}^{2}$ ). Cox regression hazards proportional models were used to examine the independent and joint association of CKD and QTc with mortality adjusting for demographic characteristics in model 1 with additional adjustment for diabetes, hypertension, history of cardiovascular disease, smoking, alcohol use, QT-prolonging medications and beta-blockers in model 2. Interaction between QTc and CKD was examined from the fully adjusted model using Wald test. We used continuous net reclassification index to test if risk stratification for mortality improves when QTc is added to the fully adjusted model with CKD. $p$ values $<0.05$ were considered statistically significant. All statistical analyses were done using Stata V.12.

\section{RESULTS}

Overall, mean age was 57 years with $54 \%$ women; $22.1 \%$ $(n=1452)$ had CKD (by either eGFR or ACR) and $14.5 \%$ $(\mathrm{n}=956)$ had prolonged QTc. Prolonged QTc was present in $12.9 \%(\mathrm{n}=658)$ of subjects without CKD compared with 20.5\% ( $\mathrm{n}=298)$ among those with CKD $(\mathrm{p}<0.0001)$. The baseline characteristics of the participants are 
Table 1 Baseline characteristics of the study population

\begin{tabular}{|c|c|c|c|c|c|c|}
\hline \multirow[t]{2}{*}{ Characteristics } & \multicolumn{3}{|c|}{ Normal QTc } & \multicolumn{3}{|c|}{ Prolonged QTc } \\
\hline & No CKD & CKD by eGFR & CKD by ACR & No CKD & CKD by eGFR & CKD by ACR \\
\hline$n$ & 4455 & 367 & 602 & 658 & 84 & 161 \\
\hline Age (years) & 54.7 & 73.4 & 61.1 & 58.7 & 74.3 & 63.3 \\
\hline African-Americans (\%) & 7.8 & 5.6 & 11.8 & 7.6 & 5.4 & 15.1 \\
\hline Female (\%) & 52.2 & 65.3 & 55.2 & 60.0 & 63.5 & 65 \\
\hline CVD (\%) & 5.2 & 18.8 & 9.7 & 13.9 & 20.4 & 16.6 \\
\hline Hypertension (\%) & 27.8 & 55.6 & 48.2 & 47.6 & 56.2 & 74 \\
\hline Diabetes (\%) & 16.1 & 63.1 & 47.6 & 31.7 & 66.2 & 52.7 \\
\hline Current smokers (\%) & 23.8 & 7.2 & 24.8 & 23.5 & 11.1 & 21 \\
\hline Alcohol use (\%) & 50.5 & 28.4 & 36.7 & 43.1 & 29.9 & 39.6 \\
\hline Body mass index $\left(\mathrm{kg} / \mathrm{m}^{2}\right)$ & 26.9 & 27.6 & 28.6 & 28.8 & 26.7 & 27.9 \\
\hline Total cholesterol & 215.8 & 228.7 & 224.6 & 218.2 & 225 & 224.4 \\
\hline Serum potassium & 4.1 & 4.2 & 4.0 & 4.0 & 4.1 & 3.9 \\
\hline Serum calcium & 9.2 & 9.4 & 9.3 & 9.2 & 9.3 & 9.1 \\
\hline $\mathrm{eGFR}\left(\mathrm{mL} / \mathrm{min} / 1.73 \mathrm{~m}^{2}\right)$ & 91.4 & 51.7 & 88.2 & 90.3 & 48.9 & 86.7 \\
\hline ACR & 7 & 10.5 & 247.7 & 9.5 & 9.7 & 292.7 \\
\hline QTc (ms) & 423 & 426.3 & 429.6 & 467.8 & 470.5 & 471.6 \\
\hline QT-prolonging medications (\%) & 4.4 & 4.5 & 6.1 & 8.0 & 3.2 & 5.0 \\
\hline Beta-blockers (\%) & 6.1 & 17.1 & 10.6 & 9.1 & 14.3 & 6.2 \\
\hline No of all deaths & 1073 & 256 & 305 & 256 & 74 & 89 \\
\hline No of CVD deaths & 419 & 134 & 131 & 99 & 39 & 44 \\
\hline
\end{tabular}

All values are expressed as mean or percent.

ACR, albumin to creatinine ratio; CKD, chronic kidney disease; CVD, cardiovascular disease; eGFR, estimated glomerular filtration rate; QTC, corrected QT.

presented in table 1 . Those with CKD are more likely to be older, have higher prevalence of cardiovascular disease, hypertension and diabetes than those without CKD. Mean cholesterol is higher in those with CKD than those without CKD. Those with CKD have longer QTc than those without CKD.

Figure 1 shows the prevalence of prolonged QTc in CKD versus no CKD stratified by race and gender. Those with CKD are more likely to have prolonged QTc compared with those without CKD for both men and women and across all races included in the study. In our linear regression analysis adjusted for demographic characteristics, history of cardiovascular disease, QT-prolonging medication and beta-blockers use, we found that those with CKD had a significantly longer QTc compared with those without CKD (mean QTc 414.1 vs $410.1 \mathrm{~ms}, \mathrm{p}=0.001$ ).

Figure 2 shows Kaplan-Meier survival estimates for all-cause and cardiovascular mortality according to CKD and QTc. For both all-cause and cardiovascular mortality, risk is higher in those with CKD than those without CKD. When further stratified by QTc, those with prolonged QTc have a much higher risk for both all-cause and cardiovascular mortality compared with normal QTc for both with and without CKD.
Median follow up was 13.7 years (maximum 18 years). Both CKD (whether by eGFR or ACR) and prolonged QTc were independently associated with all-cause and cardiovascular mortality as shown in table 2. Compared with those without CKD and normal QTc, those with CKD by eGFR only and normal QTc had a $40 \%$ increased risk of all-cause mortality (HR: $1.4(1.1-1.7))$ and $70 \%$ higher risk of cardiovascular mortality (HR: 1.7 (1.3-2.1)). If QTc was prolonged, risk was 2.6-folds higher (HR: 2.6 (1.7-3.9)) for all-cause and 3.1 folds higher (HR: 3.1 (1.7-5.4)) for cardiovascular mortality. In those with CKD by ACR, risk of both all-cause and cardiovascular mortality was higher compared with those without CKD. However, when further stratified by baseline QTc, there was no significant different between those with normal QTc compared with prolong QTc.

There was no significant multiplicative interaction between CKD (CKD by either eGFR or ACR) and prolonged QTc for all-cause or cardiovascular mortality $(\mathrm{p}=\mathrm{NS})$. However, in our risk prediction analysis, we found that there was a significant improvement in risk prediction for all-cause and cardiovascular mortality when prolong QTc was added to the fully adjusted model with CKD $(p<0.00001$ for all-cause mortality and $p<0.00001$ for cardiovascular mortality). 


\section{Prevalence of Prolonged QTc by CKD Stratified by Race and Gender}

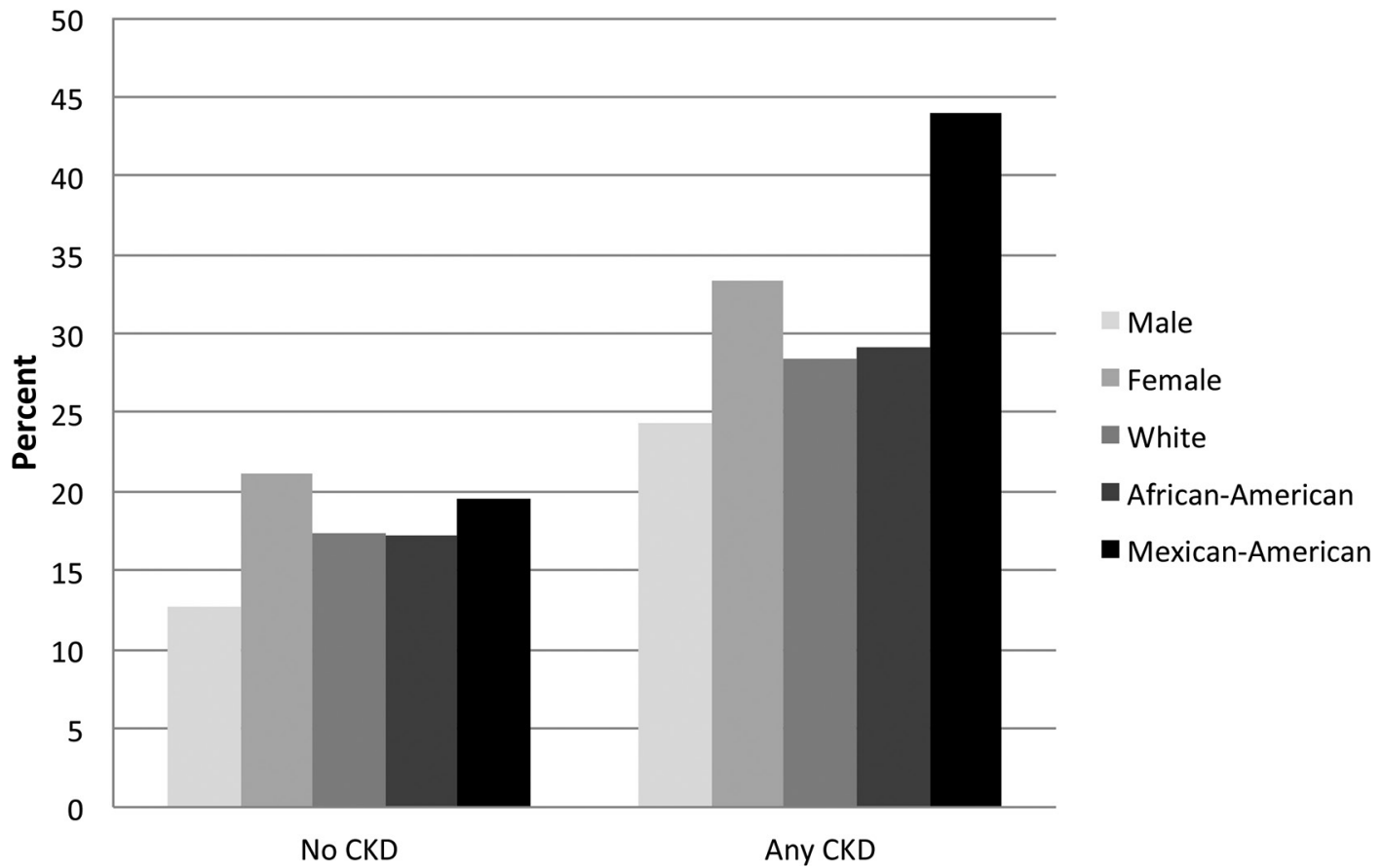

Figure 1 Prevalence of prolonged corrected QT (QTc) in the chronic kidney disease (CKD) versus no CKD subjects according to race and gender.

\section{DISCUSSION}

In this large observational study representative of the general US population, we observed that those with CKD are more likely to have prolonged QTc compared with those without CKD. This finding was consistent for both men and women and for all races included in the study. We also observed that both prolonged QTc and CKD were independently associated with increased risk of mortality. When QTc and CKD were combined, risk of mortality was higher in those with CKD by eGFR when QTc was prolonged as compared with those with normal QTc and no CKD. However, there was no significant difference in risk for either all-cause or cardiovascular mortality in those with CKD by ACR when QTc was prolonged compared with normal QTc. There was no significant multiplicative interaction between QTc and CKD for either all-cause or cardiovascular mortality. However, there was a significant improvement in risk prediction for both all-cause and cardiovascular mortality when QTc was added to CKD.

Our findings are consistent with the existing evidence that both QTc and CKD are independently associated with increased mortality risk. ${ }^{19}{ }^{20}$ A prior study done by Zhang et al on QT duration and its relationship with mortality documented that both the short and the long QT intervals were associated with increased risk of mortality. ${ }^{19}$ Other population-based studies in the

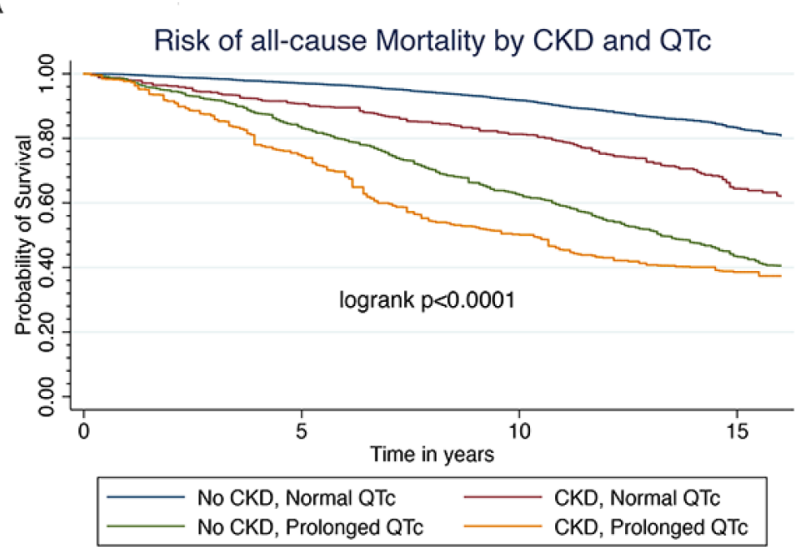

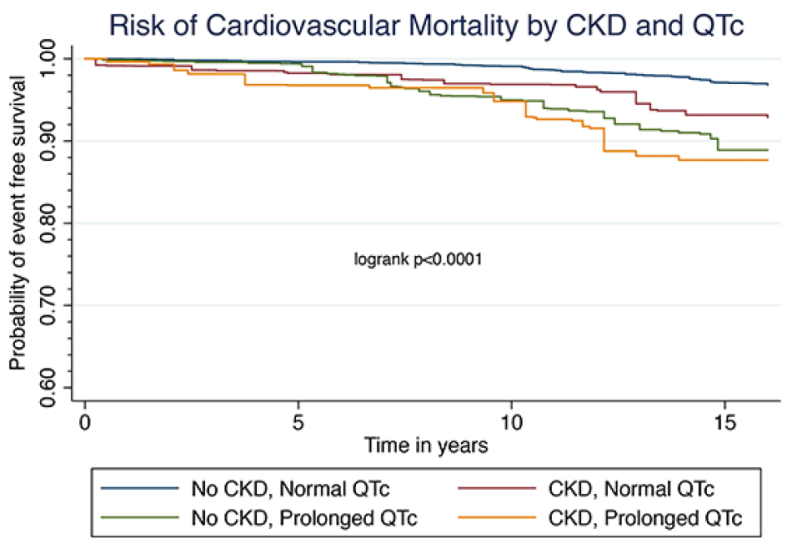

Figure 2 Kaplan-Meier survival estimates for (A) all-cause and (B) cardiovascular mortality according to corrected QT (QTc) and chronic kidney disease (CKD). 


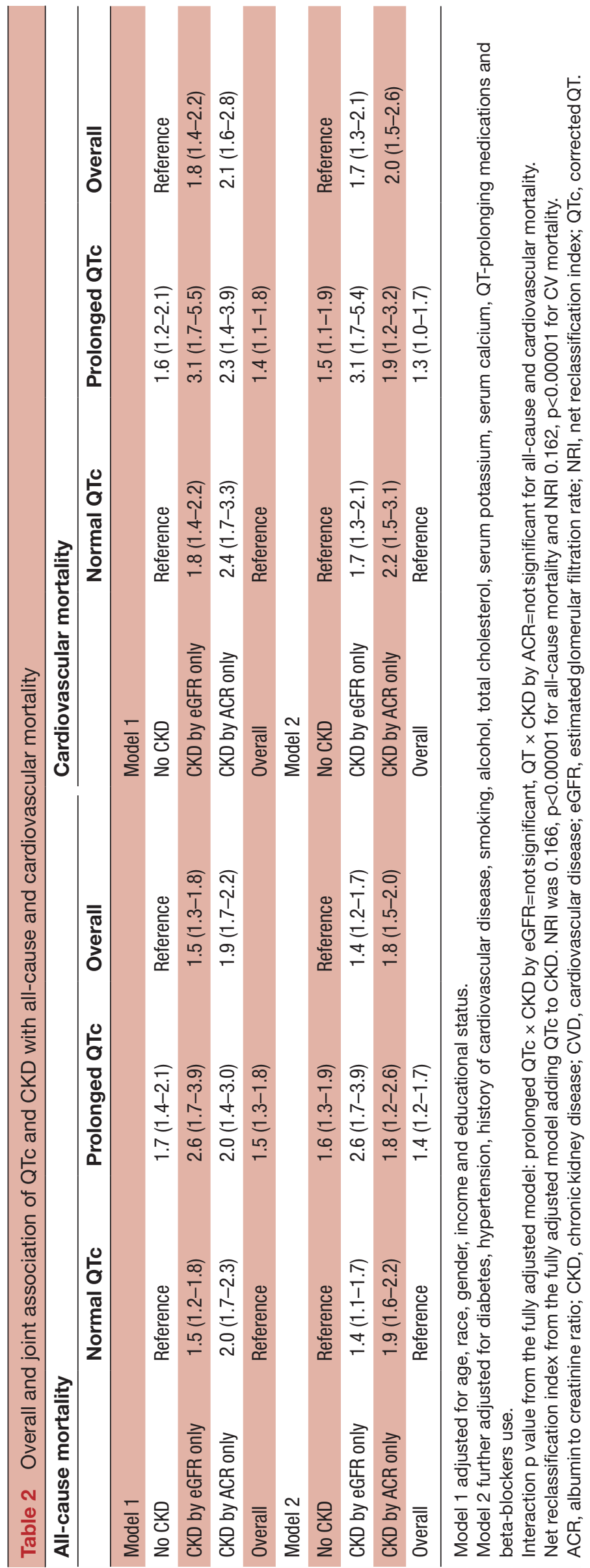


past have also shown prolonged QT intervals leading to increased overall mortality risk. ${ }^{21-23}$ Similarly, CKD is known to be attributive to high mortality and progression of cardiovascular disease. ${ }^{24} \mathrm{~A}$ systemic review with 39 studies and 1371990 participants concluded that patients with CKD are at a significantly higher risk of CVD-related mortality compared with those without CKD. ${ }^{25}$ Shafi et al documented that patients with eGFR ranging from 30 to $59 \mathrm{~mL} / \mathrm{min} / 1.73 \mathrm{~m}$ had a $\mathrm{HR}$ of 1.49 (95\% CI 1.16 to 1.92 ) for CVD associated mortality and a HR of 1.39 (95\% CI 1.17 to 1.65 ) for all-cause mortality. ${ }^{10}$ There is limited data on QTc in those with CKD especially in early stages of CKD. Furthermore, there is limited evidence on the joint association of both QTc and CKD with mortality. Data from chronic renal insufficiency cohort study (CRIC) study suggested that those with CKD and prolonged QTc have a higher risk of mortality compared with those CKD and prolonged QTc. ${ }^{26}$ However, the sample in that study only included subjects with CKD, and very few had eGFR $>60$. Our study is unique in that it represents general US population, has broad range of eGFR and a reference category that includes a large sample of subjects with normal QTc and normal kidney function. We also examined the joint association of CKD and QTc with mortality in the overall CKD sample and then further stratified to examine the joint association of albuminuria and QTc as well as eGFR and QTc with outcomes, which has not been done to our knowledge in a large representative sample. In our study, subjects with CKD by eGFR and prolonged QTc had a 2.6-folds higher mortality risk $(2.6,95 \%$ CI 1.7 to 3.9) when compared with those without CKD and normal QTc, a finding similar to the study by Hage et al. However, the population in that study was maintained on haemodialysis. ${ }^{27}$ They concluded that prolonged QTc interval was an independent predictor of mortality in end-stage renal disease regardless of age, gender, diabetes mellitus, left ventricular hypertrophy, left ventricular ejection fraction and presence of coronary artery disease on angiography. Our finding that prolonged QTc and CKD by albuminuria alone have a similar risk of mortality compared with those with normal QTc is interesting. The exact reason for this finding is not clear. It is possible that those with CKD are more likely to have electrolyte abnormalities, and this is usually true in those with abnormal kidney filtration function manifested by a decline in eGFR as compared with those with albuminuria alone with normal filtration function. These electrolyte abnormalities could be the potential reason for increased mortality in those with abnormal kidney filtration function compared with normal filtration function. Further studies are needed to examine this association.

Our findings of prolonged QTC in those with CKD have been observed in small studies. ${ }^{28}{ }^{29}$ However, this is the first study to our knowledge to report this in a large representative sample and to provide information by gender and race. In the past, the association between prolonged QTc and CKD has been said to be confounded by factors such as $\mathrm{Ca}^{2+}$ levels, albumin levels and diabetes, but a study by Kurosu et al did not find any significant evidence documenting relationship between these factors and prolonged QTc in patients with CKD. ${ }^{30}$ In a recent study by Tang et al, uraemic retention of solute indoxyl sulfate levels has been purposed to be the reason for the prolongation of the QTc in patients with CKD. It is proposed that in vitro indoxyl sulfate affects the delayed rectifier potassium channels in the cardiac myocytes by downregulating the potassium channel phosphorylation leading to decreased current activity of these channels, which causes prolonged action potential duration. Prolong action potential duration leads to prolongation of the QTc interval. ${ }^{31}$

The major strengths of our study include a large representative sample that is well established and designed for research. We also had a long follow-up in this sample. All variables were measured with high reliability. This is one of the first studies to examine the joint association of both CKD by eGFR and albuminuria and QTc with mortality in a representative sample of US population. There are some limitations of our study as well that are worth mentioning. First, we had a single automated measurement of QTc. However, prior studies have used the same measurement and have found consistent results. Automated measurement may be subject to measurement error, but those are likely to be random. Second, our study lacked data on left ventricular function and other structural abnormalities of the heart, which would have been useful.

In conclusion, QTc is longer in those with CKD compared with those without CKD for both men and women and across different races. There is a significantly higher risk of all-cause and cardiovascular mortality in those with CKD by eGFR and prolonged QTc compared with those with normal QTc and no CKD. Risk prediction for both all-cause and cardiovascular mortality improves significantly when QTc is added to CKD beyond conventional cardiovascular risk factors. Caution needs to be exercised when prescribing QT-prolonging medication and keeping electrolytes in the normal range in those with CKD when QTc is prolonged as these individuals are at a significantly higher risk of mortality compared with those with normal kidney function. A screening ECG in those with CKD may help in finer risk stratification and may be considered.

Contributors RM, SW and SEW did substantial contributions to the conception and design of the work.RM, SW and SEW work with the acquisition, analysis and interpretation of data for the work. DP and JP drafted the work and revised it critically for important intellectual content. RM, SW, SEW, JP and DP were involved in the final approval of the version to be published. RM and SW worked in agreement to be accountable for all aspects of the work in ensuring that questions related to the accuracy or integrity of any part of the work are appropriately investigated and resolved.

Competing interests None declared.

Ethics approval We used data from the National Health and Nutrition Examination Survey (NHANES). The National Center for Health Statistics of the Centers for Disease Control and Prevention institutional review board approved the protocol for NHANES. All participants gave written informed consent. 
Provenance and peer review Not commissioned; internally peer reviewed.

Data sharing statement There are no additional data available for this paper.

Open Access This is an Open Access article distributed in accordance with the Creative Commons Attribution Non Commercial (CC BY-NC 4.0) license, which permits others to distribute, remix, adapt, build upon this work non-commercially, and license their derivative works on different terms, provided the original work is properly cited and the use is non-commercial. See: http://creativecommons.org/ licenses/by-nc/4.0/

(c) Article author(s) (or their employer(s) unless otherwise stated in the text of the article) 2017. All rights reserved. No commercial use is permitted unless otherwise expressly granted.

\section{REFERENCES}

1. Coresh J, Selvin E, Stevens LA, et al. Prevalence of chronic kidney disease in the United States. JAMA 2007;298:2038.

2. Kidney Disease Statistics for the United States, National Institute of Diabetes and Digestive and Kidney Diseases (NIDDK) (cited 24 Dec 2016). https://www.niddk.nih.gov/health-information/healthstatistics/Pages/kidney-disease-statistics-united-states.aspx.

3. Fast Facts - the national kidney foundation. https://www.kidney.org/ news/newsroom/factsheets/FastFacts (cited 24 dec 2016).

4. Navaneethan SD, Schold JD, Arrigain S, et al. Cause-specific deaths in non-dialysis-dependent CKD. J Am Soc Nephrol 2015;26:2512-20.

5. Voiculescu M, Ionescu C, Ismail G. Frequency and prognostic significance of QT prolongation in chronic renal failure patients. Rom $J$ Intern Med 2006;44:407-17.

6. Zhang Y, Post WS, Dalal D, et al. QT-interval duration and mortality rate: results from the third national health and nutrition examination survey. Arch Intern Med 2011;171:1727-33.

7. Priori SG, Schwartz PJ, Napolitano C, et al. Risk stratification in the long-QT syndrome. N Engl J Med 2003;348:1866-74.

8. Sauer AJ, Moss AJ, McNitt S, et al. Long QT syndrome in adults. J Am Coll Cardiol 2007;49:329-37.

9. Zhang Y, Post WS, Blasco-Colmenares E, et al. Electrocardiographic QT interval and mortality. Epidemiology 2011;22:660-70.

10. Shafi T, Matsushita K, Selvin E, et al. Comparing the association of GFR estimated by the CKD-EPI and MDRD study equations and mortality: the third national health and nutrition examination survey (NHANES III). BMC Nephrol 2012;13:42.

11. Burt VL, Harris T. The third national health and nutrition examination survey: Contributing data on aging and health. Gerontologist 1994;34:486-90.

12. Johnson CL, Paulose-Ram R, Ogden CL, et al. National Health and Nutrition Examination Survey: analytic guidelines, 1999 to 2010. Vital Health Stat 2 2013;161:1-24.

13. Arizona Center for Education and Research on Therapeutics. http://www.dpic.org/links/arizona-center-education-and-researchtherapeutics (cited 24 Dec 2016).
14. Rautaharju PM, Maclnnis PJ, Warren JW, et al. Methodology of ECG interpretation in the Dalhousie program; NOVACODE ECG classification procedures for clinical trials and population health surveys. Methods Inf Med 1990;29:362-74.

15. Rabkin SW, Cheng XB. Nomenclature, categorization and usage of formulae to adjust QT interval for heart rate. World J Cardiol 2015;7:315-25.

16. Selvin E, Manzi J, Stevens LA, et al. Calibration of serum creatinine in the National Health and Nutrition Examination Surveys (NHANES) 1988-1994, 1999-2004. Am J Kidney Dis 2007;50:918-26.

17. Levey AS, Stevens LA, Schmid CH, et al. A new equation to estimate glomerular filtration rate. Ann Intern Med 2009;150:604-12.

18. CDC. NCHS data Linkage - Mortality Data - Public-Use Files. https:// www.cdc.gov/nchs/data-linkage/mortality-public.htm (cited 22 Feb 2017).

19. Zhang Y, Post WS, Dalal D, et al. QT-Interval Duration and Mortality Rate. Arch Intern Med 2011;171:1727-33.

20. Matsushita K, van der Velde M, Astor BC, et al. Association of estimated glomerular filtration rate and albuminuria with all-cause and cardiovascular mortality in general population cohorts: a collaborative meta-analysis. Lancet 2010;375:2073-81.

21. Sheehan J, Perry IJ, Reilly M, et al. QT dispersion, QT maximum and risk of cardiac death in the caerphilly heart study. Eur $J$ Cardiovasc Prev Rehabil 2004;11:63-8.

22. Nilsson G. QTc interval and survival in 75-year-old men and women from the general population. Europace;8:233-40.

23. Rautaharju PM, Kooperberg C, Larson JC, et al. Electrocardiographic abnormalities that predict coronary heart disease events and mortality in postmenopausal women: the women's health Initiative. Circulation 2006;113:473-80.

24. Foley RN, Parfrey PS, Sarnak MJ. Clinical epidemiology of cardiovascular disease in chronic renal disease. Am J Kidney Dis 1998;32:S112-19.

25. Tonelli M, Wiebe N, Culleton B, et al. Chronic kidney disease and mortality risk: a systematic review. J Am Soc Nephrol 2006;17:2034-47.

26. Deo R, Shou H, Soliman EZ, et al. Electrocardiographic measures and prediction of cardiovascular and noncardiovascular death in CKD. J Am Soc Nephrol 2016;27:559-69.

27. Hage FG, de Mattos AM, Khamash $\mathrm{H}$, et al. QT prolongation is an independent predictor of mortality in end-stage renal disease. Clin Cardiol 2010;33:361-6.

28. Di lorio B, Bellasi A. QT interval in CKD and haemodialysis patients. Clin Kidney J 2013;6:137-43.

29. Bignotto LH, Kallás ME, Djouki RJ, et al. Electrocardiographic findings in chronic hemodialysis patients. J Bras Nefrol 2012;34:235-42

30. Kurosu M, Ando Y, Akimoto T, et al. [Factors related to the QT prolongation in chronic renal failure]. Nihon Jinzo Gakkai Shi 1999;41:70-6.

31. Tang WH, Wang CP, Chung FM, et al. Uremic retention solute indoxyl sulfate level is associated with prolonged QTc interval in early CKD patients. PLoS One 2015;10:e0119545. 